
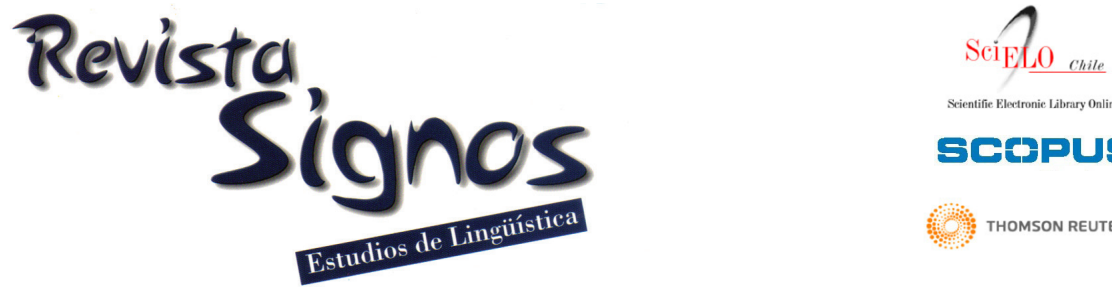

\title{
La influencia del contexto referencial en la resolución de ambigüedades oración completiva/oración de relativo: Evidencia en español*
}

\author{
The influence of referential context in solving ambiguities in \\ complete sentences/relative clause: Evidence from Spanish
}

\author{
Laura Alonso \\ laura.alonso@uam.es \\ Universidad Autónoma de Madrid \\ España
}

\author{
José Antonio León \\ joseantonio.leon@uam.es \\ Universidad Autónoma de Madrid \\ España
}

Recibido: I0-VIII-20I0 / Aceptado: I7-III-20I I

\begin{abstract}
Resumen: El presente trabajo analiza la resolución de ambigüedades sintácticas en español. De manera más específica, compara el procesamiento de oraciones con ambigüedad estructural (parsing) entre oración de relativo y oración completiva, con y sin contexto referencial, utilizando una metodología de tiempos de lectura autoadministrada. El objetivo era comprobar si los resultados encontrados en lectores de habla inglesa se obtenían también en español. Los tiempos de lectura para las frases que contenían tanto oraciones completivas como oraciones de relativo fueron significativamente menores cuando iban precedidas de un contexto referencial. Estos resultados aportan evidencia congruente con los modelos referenciales (Crain \& Steedman, 1985;Altmann \& Steedman, 1988), que apuestan por un procesamiento basado en el uso de múltiples fuentes de información a la hora de resolver ambigüedades, y no solo en información de tipo estructural, tal y como sugieren los modelos modulares (Frazier, 1987; Mitchell, I994; Mitchell, Cuetos, Corley \& Brysbaert, 1995; Cuetos, Mitchell \& Corley, 1996; Frazier \& Clifton, 1996; Carreiras \& Meseguer, 1999).
\end{abstract}

Palabras Clave: Resolución de ambigüedad sintáctica, procesamiento de oraciones, modelos referenciales, modelos modulares, tiempos de lectura, oraciones de relativo, contexto referencial, procesamiento de la lengua española. 


\begin{abstract}
This paper analyzes the resolution of syntactic ambiguities in Spanish. Specifically, it compares the processing of sentences with structural ambiguity between relative clause and complete sentences, with and without referential context, using different methods of self-administered reading times. The aim was to check whether the results found in English-speaking readers is also obtained in Spanish. The reading times for sentences containing both complete sentences and relative clauses were significantly smaller when they were preceded by a referential context. These results provide evidence consistent with referential models (Crain \& Steedman, 1985; Altmann \& Steedman, 1988) supporting a process based on the use of multiple sources of information when solving ambiguities, and not just on structural information as suggested by modular models (Frazier, 1987; Mitchell, 1994; Mitchell, Cuetos, Corley \& Brysbaert, 1995; Cuetos, Mitchell \& Corley, 1996; Frazier \& Clifton, 1996; Carreiras \& Meseguer, 1999).
\end{abstract}

Key Words: Resolution of syntactic ambiguities, sentence processing, referential models, modular models, reading times, relative clauses, referential context, processing in Spanish.

\title{
INTRODUCCIÓN
}

El lenguaje es uno de los rasgos más característicos de los seres humanos. Quizá por ello está presente, de una u otra forma, en cualquier actividad cognitiva. El conocimiento de las capacidades cognitivas sobre las que se sustenta este es parte de una empresa de mayor alcance dirigida a comprender cómo funciona la mente. Son muchos los procesos implicados en la comprensión y la producción del lenguaje, pero en el presente trabajo nos vamos a centrar en el estudio de los procesos implicados en su comprensión, más concretamente en el estudio de los mecanismos implicados en el procesamiento sintáctico de oraciones o parsing. Los estudios dedicados al análisis de los procesos que operan durante la comprensión de oraciones han encontrado en las ambigüedades sintácticas una fuente de conocimientos importante sobre los mecanismos que subyacen al procesamiento humano de oraciones. La importancia de este tipo de investigaciones radica en el hecho de que, como pone de manifiesto Pinker (1994), las ambigüedades constituyen más la norma que la excepción en cualquier idioma, tanto en el lenguaje oral como en el lenguaje escrito. Estas ambigüedades hacen referencia a los casos en los que una misma oración puede tener más de una alternativa de interpretación. Considérense, por ejemplo, las siguientes oraciones (I) y (2):

(I) El policía vio al espía con unos prismáticos.

(2) El reportero entrevistó a la hija del coronel que estuvo en Sevilla.

Ante oraciones de este tipo, el lector u oyente puede adoptar dos interpretaciones distintas. En el caso de la oración (I), puede interpretarse, por un lado, que era el policía el que tenía los prismáticos y a través de ellos vio al espía o, por el contrario, se puede considerar que la persona que portaba los prismáticos era el espía y que el policía vio a este llevando unos prismáticos. En el caso de la oración (2), el sujeto puede interpretar que era la hija la que estuvo en Sevilla o, por el contrario, que había sido el coronel. Se trata en estos casos de ambigüedades de tipo estructural o sintáctico, aunque podríamos encontrarnos con ambigüedades en muchos niveles, como las ambigüedades léxicas o de relaciones de dependencia a larga distancia. En las ambigüedades de tipo estructural el foco de la ambigüedad se establece en la estructura sintagmática de la oración, debido a que existen dos formas posibles de segmentar la oración en unidades estructurales $y$, por tanto, de establecer dependencias entre los constituyentes que la integran.

Los distintos tipos de información que se hacen disponibles durante el procesamiento de oraciones son muchos y muy variados. Estas fuentes nos dan información sobre la pronunciación, morfología, categoría léxica, estructura sintáctica, significado, puntuación y entonación o prosodia de la oración. Además, mientras leemos u oímos una oración debemos integrar estas fuentes de información con la información aportada por el contexto en el que se está produciendo la misma. El objetivo principal de las investigaciones dedicadas al estudio de la comprensión de los procesos cognitivos implicados en el procesamiento de oraciones es entender cómo se produce la combinación de dichas fuentes de información, así como analizar el momento temporal en el que cada una de ellas entra en juego durante dicho procesamiento. En este sentido, el procesador de oraciones puede ser visto como un dispositivo modular dividido en 
un conjunto de subcomponentes responsables de llevar a cabo de manera autónoma y en estadios temporales diferentes distintos aspectos centrales del procesamiento (Fodor, 1983) o como un dispositivo interactivo formado por múltiples bases de conocimiento en continua interacción y activadas de manera simultánea. Las investigaciones dedicadas al estudio del curso temporal del procesamiento de oraciones han empleado tareas de lectura controlada con registro de movimientos oculares o técnicas de lectura autoadministrada. Estas tareas permiten medir, respectivamente, el patrón de fijaciones y regresiones oculares realizadas durante la lectura y el tiempo dedicado a la lectura de cada fragmento de un texto.

\section{I.I. Modelos teóricos sobre la resolución de la ambigüedad sintáctica}

La literatura sobre este tema no es unitaria respecto a la emergencia de modelos teóricos que expliquen cómo se resuelve la ambigüedad sintáctica. De manera general, pueden dividirse en dos grupos. Los modelos basados en módulos y los modelos interactivos. Describiremos brevemente cada uno de ellos.

a) Modelos basados en módulos. Los modelos que apuestan por un procesamiento basado en módulos tales como el 'modelo de vía muerta' (Frazier, 1987), el 'modelo construal' (Frazier \& Clifton, 1996; Carreiras \& Meseguer, 1999) o el 'modelo de ajuste lingüístico' (Mitchell, 1994; Mitchell et al., 1995; Cuetos et al., 1996) coinciden en señalar que, cuando el procesador se enfrenta a una oración ambigua, recurre en un primer momento a información de tipo estructural (sobre la categoría sintáctica de las palabras y de los sintagmas junto con el conocimiento de las reglas de estructura sintagmática). Únicamente en una segunda etapa, que se produce cuando la interpretación propuesta resulta errónea cuando se compara con información léxica, semántica o discursiva, el procesador reanaliza la frase de nuevo haciendo uso de estos otros tipos de información. Cuando esto ocurre se dice que el analizador se ha metido en una vía muerta o gardenpath. De esta manera, el procesador sintáctico opera, en una primera fase, atendiendo a estrategias de carácter estructural y con independencia de las características léxicas, semánticas y pragmáticas.

Según el 'modelo de vía muerta', las estrategias estructurales que entran en juego durante el primer estadio de análisis se basan en la aplicación de dos tipos diferentes de principios o heurísticos: el principio de Adjunción Mínima ("el procesador debe optar por la estructura que postule menos nodos sintácticos potencialmente innecesarios"; Frazier \& Rayner, 1982) y el principio de Cierre Tardío ("Ios nuevos ítems de información se conectarán con el sintagma o la cláusula postulada más recientemente cuando ambos marcadores contengan el mismo número de nodos"; Frazier, I 987).

Volvamos a considerar el ejemplo (I):"El policía vio al espía con unos prismáticos".Atendiendo al principio de Adjunción Mínima, el fragmento ambiguo "con los prismáticos" se adjuntará al fragmento encabezado por el verbo "vio", lo que origina la siguiente interpretación: el policía llevaba unos prismáticos y con ellos vio al espía. Consideremos ahora el ejemplo (2): "El reportero entrevistó a la hija del coronel que estuvo en Sevilla". Desde la perspectiva de los modelos modulares, ante una oración de este tipo en la que ambos marcadores tienen el mismo número de nodos, se recurrirá al Principio de Cierre Tardío, y se interpretará la oración del siguiente modo: un coronel había estado en Sevilla y un reportero entrevistó a la hija de este. Según el modelo modular, dichas estrategias son de aplicación universal dado que obedecen a principios psicológicos generales de optimización de recursos de comprensión. El debate sobre dicha universalidad ha provocado el surgimiento de numerosos estudios empíricos en diferentes lenguas. Se ha encontrado evidencia empírica favorable sobre la aplicación del principio de Adjunción Mínima en inglés (Ferreira \& Clifton, 1986; Mitchell, Corley \& Garnham, 1992), así como en castellano (Carreiras, lgoa \& Meseguer, 1997). Sin embargo, la universalidad del principio de Cierre Tardío se ha puesto en duda en estudios en español (Mitchell, Cuetos \& Zagar, 1990; Carreiras \& Clifton, 1993; Cuetos et al., 1996; Carreiras, 1997).

b) Modelos interactivos. Por su parte, los modelos que apuestan por un procesamiento de tipo interactivo como el 'modelo de satisfacción de restricciones' (MacDonald, 1993, 1994; Trueswell, Tanenhaus \& Kello, 1993; Spivey-Knowlton \& Tanenhaus, 1994; Trueswell \& Tanenhaus, 1994; MacDonald, Pearlmutter \& Seidenberg, 1994a; MacDonald, Pearlmutter \& Seidenberg, 1994b; Trueswell, Tanenhaus \& Garnsey, 1994; Tabossi, Spivey-Knowlton, McRae \& Tanenhaus, 1994; Tanenhaus, Spivey-Knowlton \& Hanna, 2000; Trueswell, 1996, 2000) o el 'modelo referencial' 
(Altmann \& Steedman, 1988; Altmann, Garnham \& Dennis, 1992; Altmann, Garnham \& Henstra, 1994; Pickering \& Traxler, 1998; Kamide, Altmann \& Haywood, 2003) coinciden a la hora de considerar que no se debe distinguir entre una primera etapa de construcción de estructuras a partir del procesador sintáctico y una segunda etapa de evaluación y revisión a partir del procesador semántico. Por el contrario, sostienen que la información léxica, semántica y pragmática (en adelante extrasemántica) es utilizada desde el primer momento en el proceso de comprensión de oraciones. Este tipo de información permitiría, desde estadios iniciales, que se lleve a cabo una interpretación correcta de la misma.

\section{I.2. Ambigüedades oración completiva vs. oración de relativo}

Una gran cantidad de investigaciones se han basado en el análisis de oraciones que presentan la ambigüedad oración completiva/oración de relativo (o ambigüedad OC/OR). Consideremos, como ejemplo, las siguientes oraciones (3) y (4):

(3) El propietario le dijo al constructor/ que había acordado pagar/ la factura en una quincena.

(4) El propietario le dijo al constructor/ que había acordado pagar/ que la factura era muy justa.

En todas ellas aparece la cláusula "que había acordado pagar". La diferencia entre ellas reside en que, mientras que en la oración (3) dicha cláusula actúa como completiva, en la oración (4) actúa como relativo. Cuando los sujetos se enfrentan a cualquiera de las dos oraciones, deben decidir entre las dos alternativas propuestas dado que el verbo 'decir' acepta tanto cláusulas de complemento como cláusulas de relativo.

Este tipo de ambigüedad sintáctica se ha estudiado tanto en presencia como en ausencia de información extra-semántica. En ausencia de estas fuentes de información, los defensores de los modelos modulares han encontrado una preferencia de interpretación inicial del fragmento ambiguo como oración completiva cuando esta se presentaba en el vacío (sin ningún otro tipo de información desambiguadora que la puramente sintáctica o estructural). La explicación a este tipo de hallazgos se basa en el principio estructural de Adjunción Mínima. Así, Mitchell et al. (1992) llevaron a cabo una serie de experimentos para estudiar esta ambigüedad en inglés y encontraron un incremento significativo en los tiempos de lectura de la región desambiguadora de las oraciones de relativo, comparados con los tiempos medios producidos por la condición de oración completiva. Estos resultados parecían indicar que, inmediatamente después de leer el fragmento ambiguo 'que', los lectores esperaban encontrar una continuación de oración completiva. En los casos en los que el fragmento que aparecía inmediatamente después de la región ambigua no podía ser incorporado a dicha interpretación en curso, los sujetos se introducían en una vía muerta. Los trabajos desarrollados desde corrientes más interaccionistas también han encontrado una preferencia de interpretación inicial, ante este tipo de ambigüedades, como cláusula completiva. La explicación que dan es bien distinta a la anterior. En este caso, se basa en el Principio de Parsimonio:"elija el candidato disponible con las menores presuposiciones insatisfechas" (Crain \& Steddman, 1985). Así, en ausencia de un contexto en el que se explicite información sobre varios referentes, existirá una clara preferencia por la interpretación como oración completiva y no como oración de relativo asociada a uno de los referentes. Recientemente en español, Demestre y GarcíaAlbea (2004) llevaron a cabo un experimento de lectura autoadministrada para examinar si la preferencia observada en inglés también se observa en español. Los resultados de ese experimento mostraron un incremento significativo en el tiempo de procesamiento de las oraciones ambiguas que resultaban ser incompatibles con una lectura de oración completiva. La información que deshacía la ambigüedad era leída significativamente con más rapidez cuando era compatible con la interpretación de oración completiva (en adelante $\mathrm{OC}$ ) que cuando solo era compatible con la interpretación de oración de relativo (en adelante OR). Así, se mostraba una clara preferencia por interpretar inicialmente el fragmento ambiguo como una OC.

En los casos en los que se ha pretendido estudiar la influencia de la información extra-semántica sobre la resolución de ambigüedades, los resultados encontrados desde ambas perspectivas son bien distintos. Desde la perspectiva de los modelos interactivos, se ha encontrado evidencia acerca de la influencia temprana de distintos tipos de información en el procesamiento de oraciones ambiguas OC/OR (Crain \& Steedman, 1985; Altmann, 1988; Altmann et al., 1992; Altmann et al., 1994). Demestre (2003), examinó la influencia de la 
información específica de los verbos en la resolución de este tipo de ambigüedades. Encontró que los sujetos eran sensibles a la manipulación indicativo/ subjuntivo. Los resultados mostraban tiempos de lectura de las formas en indicativo significativamente mayores que los de las formas en subjuntivo. Estos datos indicaban que el sistema había accedido ya, desde etapas iniciales de análisis, a la información del verbo principal. Pero el grueso de las investigaciones han estudiado la influencia del contexto referencial en la resolución de ambigüedades OC/OR. Uno de los estudios pioneros fue llevado a cabo por Crain y Steedman (1985). Ambos autores hicieron notar que si la frase recogida al inicio (I) ("El policía vio al espía con unos prismáticos") se presenta dentro de un contexto en el que se introducían varios espías (siendo uno de ellos el que llevaba unos prismáticos), la posibilidad de interpretar 'con unos prismáticos' como instrumento de la acción denotada por 'ver' parece más remota. Altmann y Steedman (1988) encontraron resultados similares a los de Crain utilizando una metodología de tiempos de lectura. Consideremos las siguientes frases y contextos:

Frases:

a) El psicólogo le dijo a la mujer que le había preocupado por sus problemas de pareja (cláusula completiva).

b) El psicólogo le dijo a la mujer que le había preocupado que le visitara de nuevo (cláusula de relativo).

\section{Contextos:}

I) Un psicólogo estaba aconsejando a un hombre y a una mujer. Estaba preocupado por uno de los miembros de la pareja, pero no por el otro.

2) Un psicólogo estaba aconsejando a dos esposas. Estaba preocupado por una de ellas, pero no lo estaba por la otra.

En concreto, observaron que las diferencias (en tiempos de lectura) encontradas durante el procesamiento del fragmento ambiguo en las frases (a) y (b) desaparecían cuando la oración (b) iba precedida de un contexto en el que aparecían dos referentes (2), siendo uno solo de ellos el que recibía la acción. Así, las medidas en curso de los tiempos de lectura de las regiones desambiguadoras de las oraciones no presentaban diferencias significativas cuando se introducía un contexto referencial, lo que parecían mostrar que se accedía rápidamente a la información contenida en este. Más recientemente, Altmann et al.(1992) monitorizaron los movimientos oculares de los sujetos mientras leían oraciones con fragmentos ambiguos pertenecientes a cláusulas de complemento y a cláusulas de relativo. Encontraron que un contexto con dos referentes provocaba, durante la lectura de la cláusula de complemento, menores tiempos de fijación durante el primer análisis así como un menor número de regresiones en un momento posterior. Estos resultados les permitieron concluir que el contexto referencial facilitaba la interpretación del fragmento ambiguo como cláusula de relativo desde un primer estadio de análisis. Spivey-Knowlton et al. (1993) examinaron cómo ciertos tipos de información semántica y contextos referenciales de discurso afectaban al procesamiento de cláusulas de relativo que eran temporalmente ambiguas entre cláusulas de relativo y cláusulas completivas. Estos autores encontraron resultados que apoyaban la hipótesis de que el contexto puede ejercer su influencia durante las primeras etapas del procesamiento.

Pero no todos los estudios desarrollados dentro del ámbito del contexto referencial han encontrado datos a favor de la influencia inmediata de dicho contexto en la resolución de este tipo de ambigüedades. Ferreira y Clifton (1986) monitorizaron los movimientos oculares de los sujetos durante la lectura de oraciones con cláusulas de relativo reducidas en las que habían manipulado el contexto referencial (con uno o dos referentes) sin encontrar una influencia inmediata de dicho contexto durante el procesamiento. Britt, Perfetti, Garrod y Raynerja (1992) también manipularon el contexto referencial con cláusulas de relativo reducidas y encontraron que el efecto del contexto no reducía las diferencias entre el procesamiento de las cláusulas de relativo y las cláusulas principales.

En español no se ha realizado hasta la fecha un estudio que se enmarque dentro de esta línea de investigación centrada en examinar la influencia del contexto referencial durante el procesamiento de oraciones que presentan la ambigüedad OC/OR. El grueso de la investigación se ha desarrollado dentro del ámbito del habla inglesa. Dadas las enormes diferencias existentes entre las diferentes lenguas, el objetivo del presente estudio se centra en comprobar si los resultados encontrados en los lectores de habla inglesa se obtienen también en lectores del español. 


\subsection{Objetivo e hipótesis}

El presente trabajo examina el proceso de comprensión de oraciones en la lengua castellana, concretamente, el proceso de resolución de ambigüedades sintácticas. El objetivo principal es determinar el momento en el que la información semántica, más concretamente el contexto referencial, ejerce su influencia durante el procesamiento de oraciones temporalmente ambiguas entre cláusulas de relativo y cláusulas completivas.

Las hipótesis que pretendíamos contrastar a través de este experimento fueron las siguientes:

- Los sujetos interpretarán las frases ambiguas (tanto de complemento como de relativo) sin contexto referencial como cláusulas de complemento. Esto se traducirá en un mayor tiempo de lectura de la cláusula desambiguadora en las oraciones con cláusula de relativo en comparación con las de complemento. También se traducirá en tiempos de lectura similares entre las cláusulas de complemento ambiguas y las frases control (sin ambigüedad).

- Cuando se incorpore un contexto referencial que ayude a desambiguar la frase, las diferencias anteriores desaparecerán.

\section{I.2.2. Método}

Participantes. Setenta y seis estudiantes de la Licenciatura de Psicología de la Universidad Autónoma de Madrid participaron en el experimento como requisito de las prácticas de la asignatura Adquisición del conocimiento. Todos los participantes eran hablantes nativos del español. Los participantes fueron asignados al azar a uno de los tres grupos experimentales.

Materiales. El proceso de construcción de los materiales fue el siguiente. Todos los textos experimentales procedían de una reformulación de las oraciones utilizadas por Altmann et al. (1992) en su estudio sobre la influencia del contexto referencial para la eliminación del efecto de vía muerta. Dicha reformulación venía dada por la necesidad de dotar de sentido, tanto desde el punto de vista metodológico como lingüístico, a las oraciones una vez traducidas al castellano. Por ejemplo, a la hora de crear las oraciones control, fue necesario utilizar verbos que no aceptasen más que la interpretación de la región ambigua como cláusula de relativo, y dado que al traducir al castellano algunos verbos aceptaban tanto la interpretación como cláusula de relativo como la interpretación como cláusula de complemento, fue necesario modificarlos.

Todas las oraciones tenían la siguiente estructura: SN-V-SP-QUE (que en algunos casos introducía una $O C$ y en otros una OR). Se construyeron en total tres listas de estímulos:

- La primera contenía oraciones que intercalaban frases con cláusulas de complemento y cláusulas de relativo temporalmente ambiguas sin contexto. Un total de 32 frases experimentales y 15 de relleno fueron presentadas a los sujetos.

- La segunda introducía las mismas oraciones anteriores, pero con la presencia de contextos referenciales. $\mathrm{Al}$ igual que en el caso anterior, se presentaron 32 frases experimentales y 15 de relleno, cada una de ellas precedida por su correspondiente contexto.

- Por último, la tercera contenía 32 oraciones sin contexto y sin ambigüedad, y las mismas 15 oraciones de relleno de los dos casos anteriores.

Diseño. Se empleó un diseño factorial mixto $2 \times$ 2 , con un factor inter-sujeto (contexto) y un factor intra-sujeto (tipo de cláusula). El factor contexto se refería a la existencia o no de un contexto referencial previo a la presentación de las frases. El factor tipo de cláusula se refería a la presentación de cláusulas ambiguas de relativo o completivas. De esta manera, se obtenían 2 grupos experimentales:

Grupo I: frases con cláusulas ambiguas de complemento y de relativo sin contexto.

Grupo 2: frases con cláusulas ambiguas de complemento y de relativo con contexto.

Se añadió además un grupo control al que se le presentaron cláusulas de relativo similares a las experimentales, pero sin ambigüedad. Se optó, además, por introducir la variable tipo de cláusula como una variable intrasujeto con el objetivo de que los sujetos no reconociesen inmediatamente, tras la presentación de varias frases, la estructura de las mismas. Dicho de otro modo, si todas las frases presentadas a un mismo sujeto siguiesen una estructura idéntica, se podría correr el riesgo de que los sujetos reconociesen dicha estructura y resolvieran la ambigüedad como efecto de la práctica. 
Procedimiento. A cada sujeto se le presentó 47 frases, 32 eran experimentales y 15 eran de relleno con estructuras sintácticas ambiguas diferentes a las experimentales. Las frases de relleno se entremezclaron con las experimentales para evitar que los sujetos emitieran una respuesta automática y constante. Después de cada frase, tanto experimental como de relleno, aparecía una pregunta a la que los sujetos debían responder con un sí o un no. El objetivo de esta pregunta era que los sujetos prestasen atención durante la lectura de los textos.

Las frases se presentaron en un ordenador a través del programa D-Master basado en el paradigma de lectura autoadministrada. Las oraciones se presentaron divididas en cuatro exposiciones: cláusula principal, cláusula ambigua, principio de la cláusula desambiguadora y final de la cláusula desambiguadora. En los casos en los que aparecía un contexto previo a las oraciones, estos también se dividieron en tres exposiciones con el objetivo de no sobrecargar a los sujetos de estímulos visuales. Los sujetos se autoadministraban la lectura de las frases presionando una tecla. Cuando pulsaban por primera vez la tecla aparecía en la pantalla el aviso "VA A COMENZAR UNA FRASE". Cuando pulsaban de nuevo la tecla aparecía la primera exposición (cláusula principal); después de otra pulsación la segunda (cláusula ambigua), después la cuarta (comienzo de la cláusula desambiguadora) y, finalmente, la tercera (final de la cláusula desambiguadora). El ordenador registraba el tiempo trascurrido entre pulsaciones a la tecla, por lo que permitía obtener los tiempos de lectura de cada una de las exposiciones. El interés se centraba en el análisis de los tiempos de lectura de la tercera exposición (principio de la cláusula desambiguadora) dado que a través de la medición de dichos tiempos se podía observar si los sujetos se habían metido o no en una vía muerta. La división de la cláusula desambiguadora en dos exposiciones obedecía a la necesidad de equilibrar la carga de las exposiciones que, posteriormente, iban a ser objeto de análisis. Por ello, este fragmento contenía artículos, preposiciones o adverbios. Consideremos el ejemplo (I):

\section{Oración completiva:}

Le dijo a la chica que le hacía tener miedo por su actitud hostil hacia él

\section{Oración de relativo:}

Le dijo a la chica que le hacía tener miedo que le dejase tranquilo

\section{Oración neutra:}

Llamó a la chica que le hacía tener miedo y le pidió que se disculpase

En cuanto a los contextos, aquellos que precedían a $O C$ incluían un único referente. Por el contrario, en aquellos que precedían a frases con OR aparecían dos referentes. Consideremos el ejemplo (2):

\section{Contexto con un referente (previo a una} oración completiva):

Un chico joven había sido intimidado por un chico mayor y una chica en el colegio

Había intentado ser valiente, pero no había podido evitar las lágrimas

Tenía miedo de la chica, pero no estaba demasiado preocupado por el chico

\section{Oración de relativo:}

Un chico joven había sido intimidado por dos chicas en el colegio

Había intentado ser valiente, pero no había podido evitar las lágrimas

Tenía miedo de una de las chicas, pero no estaba demasiado preocupado por la otra

\section{Resultados}

Para las tres condiciones experimentales se calcularon los tiempos de lectura de la tercera exposición, correspondiente al inicio del fragmento que contenía información desambiguadora. La Tabla I muestra los estadísticos descriptivos referidos a los tiempos medios de lectura de los tres niveles de la variable tipo de cláusula (completiva, de relativo y neutra) en cada uno de los niveles de la variable contexto (presencia y o presencia de contexto referencial).Téngase en cuenta que el nivel neutra de la variable tipo de cláusula solo se ha administrado sin presencia de contexto, dado que era en el único nivel de la variable contexto importante para nuestras hipótesis iniciales. 
Tabla I. Estadísticos descriptivos relativos a los tiempos medios de lectura (en mls) de los tres niveles de la variable tipo de cláusula (completiva, de relativo y neutra) y en cada uno de los niveles de la variable contexto (presencia y o presencia de contexto referencial).

\begin{tabular}{|l|l|r|c|c|c|r|}
\hline Contexto & Tipo de cláusula & N & Mínimo & Máximo & Media & \multicolumn{1}{c|}{ Desv. típ } \\
\hline Con contexto & Relativo & 25 & 527,09 & 1283,20 & 779,30 & 188,36 \\
& Completiva & 25 & 468,29 & 1135,75 & 723,40 & 164,43 \\
& Neutra & 25 & 352,50 & 582,50 & 735,71 & 58,82 \\
\hline Sin contexto & Relativo & 26 & 370,94 & 869,10 & 606,52 & 117,69 \\
& Completiva & 26 & 348,74 & 711,48 & 535,57 & 96,63 \\
& Neutra & 0 & & & & \\
\hline
\end{tabular}

Se efectuó un ANOVA factorial con medidas repetidas en un factor basado en las medias calculadas para las frases que contenían una OC y una OR. La Tabla 2 muestra los estadísticos $F$ univariados referidos a los efectos intra-sujetos. Todos ellos permiten contrastar las hipótesis nulas referidas a los efectos en los que se encuentra involucrado el factor intrasujetos tipo de cláusula, a saber, el propio factor y la interacción entre el tipo de cláusula y el contexto. Los cuatro estadísticos coinciden en señalar que el efecto del factor tipo de cláusula es significativo $(p<0,05)$. Así, la cantidad de tiempo no es la misma en las dos condiciones definidas por dicho factor. Pero no ocurre lo mismo con la interacción tipo de cláusula * contexto $(P=0,63)$.
En relación al factor intersujetos contexto, el nivel crítico asociado al estadístico $\mathrm{F}(22,83, \mathrm{p}<0,00 \mathrm{I})$ permite rechazar la hipótesis nula y afirmar que el efecto del factor contexto es significativo. Puede concluirse así que la cantidad de tiempo de lectura no es la misma que las dos condiciones definidas por dicho factor. Por otra parte, la información aportada por el estadístico $\mathrm{F}$ de Levene permite concluir que, solo para el caso de las frases con OR $(p>0,05)$ puede asumirse que las varianzas de las dos poblaciones definidas por el factor contexto son iguales. La Tabla 3 recoge el resultado de las comparaciones por pares entre los niveles del factor tipo de cláusula. El resultado de estas comparaciones indica que la cantidad de tiempo consumido es significativamente mayor cuando las frases incorporan una OR que cuando incorporan una OC.

Tabla 2. Pruebas de efectos intra-sujetos (tipo de cláusula y tipo de cláusula * contexto).

\begin{tabular}{|ll|c|c|}
\hline Efecto & & F & Significación \\
\hline Tipo de cláusula & Esfericidad asumida & 16,67 &, 00 \\
& Greenhouse- Geisser & 16,67 &, 00 \\
& Huynh- Feldt & 16,67 &, 00 \\
& Límite-inferior & 16,67 &, 00 \\
\hline Tipo de cláusula* & Esfericidad asumida &, 24 &, 63 \\
Contexto & Greenhouse- Geisser &, 24 &, 63 \\
& Huynh- Feldt &, 24 &, 63 \\
& Límite-inferior &, 24 &, 63 \\
\hline
\end{tabular}

Tabla 3. Comparaciones por pares tipo de cláusula.

\begin{tabular}{|cl|c|c|}
\hline (I) Tipo de cláusula & $(\mathrm{J})$ Tipo de cláusula & Diferencia entre medias (I-J) & Significación \\
\hline Relativo & Complemento & 63,43 &, 00 \\
\hline Complemento & Relativo & $-63,43$ &, 00 \\
\hline
\end{tabular}


La Tabla 4 recoge el resultado de comparar por pares los niveles del factor contexto dentro de cada nivel del factor tipo de cláusula. Tanto en los casos en los que se introduce una OC como una OR, los tiempos de lectura son mayores sin la presencia de un contexto previo, que con la presencia de este $(p<0,00$ I en todos los casos). La Figura I contiene un gráfico de perfil representando el efecto de la interacción tipo de cláusula * contexto.

Por último, se efectuó un contraste sobre las medias obtenidas en las frases con oraciones completivas y con oraciones neutras tomando únicamente el nivel sin presencia de contexto mediante el procedimiento prueba $T$ para muestras independientes. La Tabla 5 ofrece, en primer lugar, el contraste de Levene (F) sobre homogeneidad de varianzas. Dado que la probabilidad asociada es muy pequeña $(p=0,0 l)$, debemos rechazar la hipótesis de igualdad de varianzas. No asumiendo varianzas iguales, el estadístico t toma el valor $-4,95$ y tiene asociado un nivel crítico bilateral menor que $p<0,001$. De esta manera, se puede afirmar que los datos muestrales son incompatibles con la hipótesis nula de igualdad de medias. El tiempo de lectura de las oraciones completivas fue mayor que el de las oraciones neutras.

Tabla 4. Comparaciones por pares tipo de cláusula * contexto.

\begin{tabular}{|l|c|c|c|c|}
\hline Tipo de cláusula & \multicolumn{2}{|c|}{ Presencia contexto referencial } & Diferencias & Significación \\
\hline Relativo & Sí & No & $-172,78$ &, 00 \\
\hline Complemento & Sí & No & $-187,83$ &, 00 \\
\hline
\end{tabular}

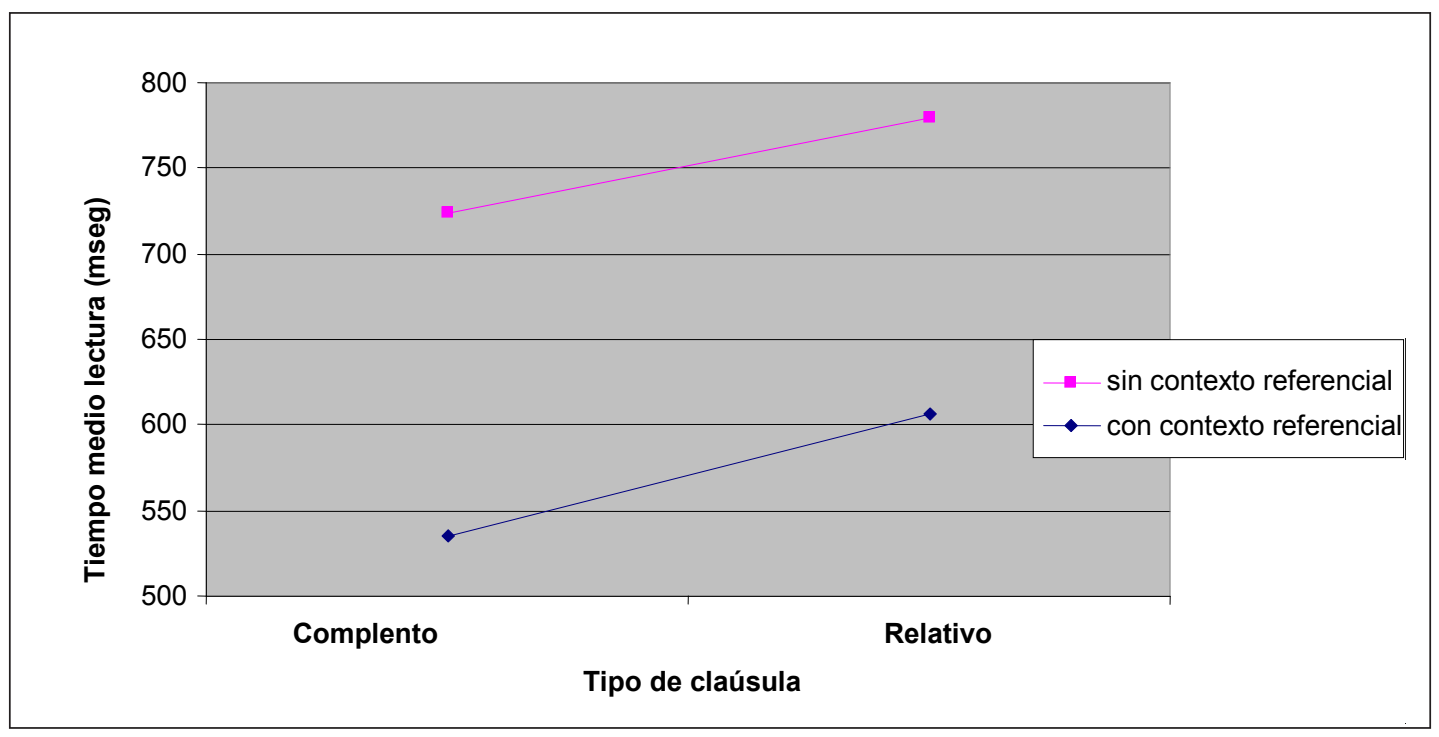

Figura I. Gráfico de perfil del efecto de la interacción tipo de cláusula * contexto.

Tabla 5. Prueba de muestras independientes.

\begin{tabular}{|l|c|c|c|c|}
\cline { 2 - 5 } \multicolumn{1}{c|}{} & \multicolumn{2}{c|}{ Prueba de Levene } & \multicolumn{2}{c|}{ Prueba T } \\
\cline { 2 - 5 } \multicolumn{1}{c|}{} & F & Significación & t & Significación \\
\hline Se han asumido varianzas iguales & 7,56 &, 01 & & \\
\hline No se han asumido varianzas iguales & & & $-4,95$, & 00 \\
\hline
\end{tabular}




\section{DISCUSIÓN}

Los resultados de la región desambiguadora muestras que los sujetos son sensibles a la introducción de un contexto referencial. El patrón de tiempos de lectura en esta región es significativamente menor ante la presencia de un contexto referencial previo a la oración que cuando esta se presenta de manera aislada, lo que sugiere que los sujetos son sensibles a la información detallada en el mismo. Esta influencia se produce tanto en el caso de las frases con oraciones completivas como con oraciones de relativo.

Los datos obtenidos en la región desambiguadora atendiendo a la variable tipo de cláusula son difíciles de explicar desde el modelo de vía muerta. Los resultados del ANOVA mostraron diferencias significativas entre las oraciones que contenían OC y OR, tanto en presencia de contexto referencial como en ausencia del mismo. En concreto, los tiempos de lectura en los dos niveles de la variable contexto, fueron mayores en el caso de las oraciones de relativo que en las completivas. Este resultado indicaría que, tal y como postulan los modelos modulares, los sujetos habrían construido inicialmente la estructura de la oración completiva basándose en principios puramente estructurales. En los casos en los que se hubieran encontrado con un fragmento desambiguador incompatible con esta estructura inicial, habrían llevado a cabo un proceso de reevaluación que explicaría los mayores tiempos de lectura de dicha región cuando se trataba de frases que contenían oraciones de relativo. Sin embargo, los modelos modulares tendrían dificultades para explicar el hecho de que los tiempos de lectura de las frases que contienen oraciones completivas fueron mayores cuando se produjeron en el vacío que cuando se encontraban inmersas dentro de un contexto. La diferencia entre los tiempos medios de lectura resultó significativa. Aún asumiendo que se ha producido un rápido acceso inicial a la información de tipo estructural, se podría considerar que los sujetos fueron sensibles a la información contenida en el contexto incluso desde estadios iniciales de procesamiento. Los modelos modulares también tendrían dificultades para explicar los datos obtenidos al comparar los tiempos de lectura de la región desambiguadora en las frases que contenían oraciones completivas con los tiempos de lectura de la correspondiente región en las frases neutras. La Prueba T para muestras independientes mostró diferencias significativas entre ambas medias. Los resultados indicaron un mayor tiempo de lectura en el caso de las oraciones completivas. De esta manera, el uso de la información de tipo sintáctico ante la presencia de ambigüedades no permite construir inicialmente la estructura de la OC basándose en principios puramente estructurales tan rápidamente como cabría esperar desde los modelos modulares. Las diferencias encontradas entre las oraciones completivas y las neutras indican que, las primeras, requieren un mayor esfuerzo cognitivo por parte de los sujetos. El sistema es capaz de detectar la ambigüedad cuando esta aparece y necesita consultar los tipos de información disponibles para desambiguarla.

Los resultados parecen más fáciles de explicar desde un enfoque interactivo. Así, cuando los sujetos se encuentran ante una oración ambigua, hacen uso de manera temporalmente paralela, de todas las fuentes de información que tienen disponible para desambiguarla. De este modo, y para el caso que nos ocupa, cuando se encuentran con una frase que presenta una ambigüedad OC/OR, no solo utilizan la información puramente estructural contenida en la oración, sino también la información detallada en el contexto referencial que se presenta previo a esta. En ausencia de contexto, los sujetos atienden al único tipo de información disponible, a saber, información de tipo sintáctica. En base a esta fuente de conocimientos, extraen una representación de la frase siguiendo el camino de la OC. Cuando los sujetos llegan al fragmento desambiguador continúan por este camino (si es compatible con la representación inicial) o llevan a cabo un proceso de reevaluación (si la alternativa escogida es incompatible con dicha representación y, por lo tanto, han seguido un camino de vía muerta). En presencia de contexto, los sujetos tienen disponible dos tipos de información, una de tipo sintáctico y otra de tipo contextual. El uso simultáneo de ambos tipos de información puede explicar el hecho de que, aún existiendo diferencias en los tiempos de lectura entre la OC y la OR, estos sean menores, en ambos casos, cuando la frase se presenta ligada a un contexto previo. Cuando la frase incorpora una OC, la alternativa de los sujetos siguiendo el camino de cierre tardío se ve reforzada por la información contextual. En los casos en los que la frase incorpora una OR la activación de ambos tipos de información puede llevar a un proceso de competición entre las dos alternativas que podría traducirse en un mayor 
tiempo de lectura que en el caso de las OC. No se trata, por tanto, de que los sujetos se hayan metido en una vía muerta, tal y como ocurría en ausencia de contexto, sino más bien, de que se ha producido un proceso de competición debido a la incompatibilidad de la información aportada por las distintas fuentes. En todo caso, en presencia de contexto, y a pesar de este proceso de competición, la ambigüedad se resuelve más rápidamente que en los casos en los que el contexto no está disponible. De esta manera, parece que el contexto referencial deviene accesible desde estadios iniciales de procesamiento.

Se podría argumentar que la técnica empleada, a saber, el registro de los tiempos de lectura, no es lo suficientemente sensible como para poder examinar si la influencia que ejerce el contexto opera inmediatamente o si dicha fuente de información se usa para revisar las decisiones tomadas siguiendo primero una estrategia estructural de cierre tardío. Sin embargo, la presentación de cada oración en cuatro exposiciones, nos permitió obtener los tiempos ( $y$, por tanto, una aproximación al esfuerzo cognitivo requerido) que dedicaban los sujetos a la lectura del fragmento que contenía el primer contacto con la información que habría de ser compatible o incompatible con un cierre tardío. El tiempo de lectura de dicha exposición permitió examinar lo que estaba computando el sistema en tiempo real y sin posibilidades de reevaluación posterior.

Los resultados obtenidos permiten aportar datos al debate entre las concepciones modulares e interactivas. La influencia inmediata del contexto sobre las decisiones que lleva a cabo el procesador sintáctico cuando se enfrenta a frases con cierto grado de ambigüedad (como es el caso de las ambigüedades OC/OR) permite suponer que nos encontramos ante un dispositivo interactivo formado por gran cantidad de fuentes de conocimiento en continua interacción y activadas de manera simultánea, más que como un dispositivo modular que opera de manera autónoma. La importancia de estos resultados se extiende más allá del simple análisis de la conducta de los sujetos ( $y$, por tanto, del procesador sintáctico) ante un tipo determinado de ambigüedad, aporta conocimiento sobre el proceso de comprensión de oraciones $y$, de manera más amplia, sobre las bases del lenguaje humano. Podemos destacar, a este respecto, el estudio llevado a cabo por Grodner, Gibson y Watson (2003) en el que estudiaron la influencia del contexto referencial en la interpretación de oraciones no ambiguas. Utilizando frases que contenían cláusulas restrictivas (sin comas) y no restrictivas (con comas) observaron que el contexto referencial facilitaba el procesamiento de oraciones restrictivas. Las oraciones a las que nos enfrentamos los lectores en nuestro día a día no se producen en el vacío sino que van inmersas en contextos discursivos que nos ayudan a un correcto procesamiento. Parece que tal contexto es utilizado, no solo como pista para la resolución de cuantas ambigüedades puedan existir en el lenguaje, sino también para la correcta interpretación de aquellas oraciones que no presentan ningún tipo de ambigüedad. Sin embargo, el debate continúa abierto y se hace indispensable el surgimiento de nuevos estudios que ayuden a dilucidar la universalidad o no del uso de este tipo de información contextual desde etapas iniciales de procesamiento en diferentes lenguas $y$ ante diferentes tipos de ambigüedades. 


\section{REFERENCIAS BIBLIOGRÁFICAS}

Altmann, G. (1988). Ambiguity, parsing strategies, and computational models. Language and Cognitive Processes, 3(2), 73-97.

Altmann, G. \& Steedman, M. (1988). Interaction with context during human sentence processing. Cognition, 30(3), 191-238.

Altmann, G., Garnham, A. \& Dennis,Y.(1992).Avoiding the garden path: Eye movements in context. Journal of Memory and Language, 3I, 685-7I2.

Altmann, G., Garnham, A. \& Henstra,J. (1994). Effects of syntax in human sentence parsing: Evidence against a structure-based proposal mechanism. Journal of Experimental Psychology: Learning, Memory and Cognition, 20, 209-2I6.

Brit, M., Perfetti, C., Garrod, S. \& Rayner, K. (1992). Parsing and discourse: Context effects and their limits. Journal of Memory and Language, 31, 293- 314.

Carreiras, M. (1997). Descubriendo y procesando el lenguaje. Madrid:Trotta.

Carreiras, M. \& Clifton, C. (1993). Relative clause interpretation preferences in Spanish and English. Language and Speech, 36, 353-372.

Carreiras, M., Igoa, J. M. \& Meseguer, E. (1997). On the use of minimal attachment strategy in Spanish: An eyetracking study. En Poster presentado en la IOth annual CUNY sentence processing conference. Los Angeles, CA, USA.

Carreiras, M. \& Meseguer, E. (1999). Procesamiento de oraciones ambigüas. En M. De Vega \& F. Cuetos (Eds.), Psicolingüística del español (pp. I63- 203). Madrid:Trotta.

Crain, S. \& Steedman, M. (1985). On not being led up the garden path:The use of context by the psychological parser. En D. Dowty, L. Karttunen \& A. Zwicky (Eds.), Natural language parsing: Psychological, computational, and theoretical perspectives (pp. 320-358). Nueva York: Cambridge University Press.

Cuetos, F., Mitchell, D. \& Corley, M. ( 1996). Parsing in different languages. En M. Carreiras, J. GarcíaAlbea \& N.Sebastián (Eds.), Language processing in Spanish (pp. I45- I87). Hillsdale, NJ:Earlbaum.

Demestre,J. (2003). Procesamiento de frases e información léxica.Tesis de Doctorado para la obtención del título de Doctor en Psicología, Departamento de Psicología, Universitat Rovira i Virgili, Cataluña, España.

Demestre, J. \& García-Albea, J. (2004). The on-line resolution of the sentence complement/relative clause ambiguity: Evidence from Spanish. Experimental Psychology, 5 I (I), 59-7I.

Ferreira, F. \& Clifton, C. (1986). The independence of syntactic processing. Journal of Memory and Language, 25, 348-368.

Fodor, J. (1983). The modularity of mind. Cambridge, MA: MIT Press.

Frazier, L. (1987). Sentence processing: A tutorial review. En M. Coltheart (Ed.), Attention and Performance XII (pp. 56I-587). Hillsdale, NJ: Erlbaum.

Frazier, L. \& Rayner, K. (1982). Making and correcting errors during sentence comprehension: Eye movements in the analysis of structurally ambiguous sentences. Cognitive Psychology, I4, I78210.

Frazier, L. \& Clifton, C. (1996). Construal. Cambridge, MA: MIT Press.

Grodner, D., Gibson, E. \& Watson, D. (2003). The influence of contextual on syntactic processing: Evidence for strong- interaction in sentences comprehension. Cognition, 95, 275-296. 
Kamide,Y., Altmann, G.\& Haywood,S. (2003).The time-course of prediction in incremental sentence processing: Evidence from anticipatory eye-movements. Journal of Memory and Language, 49, $133-156$.

MacDonald, M. C.,(1993). The interaction of lexical and syntactic ambiguity. Journal of Memory and Language, 32, 692-7I5.

MacDonald, M. C.,(1994). Probabilistic constraints and syntactic ambiguity resolution. Language and Cognitive Processes, 9, 157-20I.

MacDonald, M. C., Pearlmutter, N. \& Seidenberg, M. (1994a). The lexical nature of syntactic ambiguity resolution. Psychological Review, I01, 676-703.

MacDonald, M. C., Pearlmutter, N. \& Seidenberg, M. (1994b). Syntactic ambiguity resolution as lexical ambiguity resolution. En C. Clifton, L. Frazier \& K. Rayner (Eds.), Perspectives on sentence processing (Pp. 123-153). Hillsdale, NJ: Erlbaum.

Mitchell, D. (1994). Sentence parsing. En M.A. Gernsbacher (Ed.), Handbook of psycholinguistics (pp. 375-409). San Diego:Academic Press.

Mitchell, D., Cuetos, F. \& Zagar, D. (1990). Reading in diferent languages: Is there a universal mechanism for parsing sentences? En D. Balota, G. Flores d'Arcais \& K. Rayner (Eds.), Comprehension processes in reading (pp. 285-302). Hillsdale, NJ: Erlbaum.

Mitchell, D., Corley, M. \& Garnham, A. (1992). Effects of context inhuman sentence parsing: Evidence against a discourse-based proposal mechanism. Journal of Experimental Psychology: Learning, Memory and Cognition, 18, 69-88.

Mitchell, D., Cuetos, F., Corley, M. \& Brysbaert, M. (1995) Exposure-based models of human parsing: Evidence for the use of coarse-grained (non-lexical) statistical records. Journal of Psycholinguistic Research, 24, 469-488.

Pinker, S. (1994). The language instinct. How the mind creates languages. New York: Harper Collins.

Pickering, M. \& Traxler, M. (1998). Plausibility and recovery from garden paths: An eye-tracking study.Journal of Experimental Psychology: Learning, Memory, and Cognition, 24, 940-96I.

Spivey-Knowlton, M., Trueswell, J. \& Tanenhaus, M. (1993). Context effects in syntactic ambiguity resolution: Parsing reduced relative clauses. Canadian Journal of Psychology: Special Issue: Reading and Language Processing, 47(2), 276-309.

Spivey-Knowlton, M. \& Tanenhaus, M. (1994). Referential context and syntactic ambiguity resolution. En C. Clifton, L. Frazier \& K. Rayner (Eds.), Perspectives on sentence processing (pp. I55- I80). Hillsdale, NJ: Erlbaum.

Tabossi, P., Spivey-Knowlton, M., McRae, K. \& Tanenhaus, M. (I994). Semantic effects on syntactic ambiguity resolution. En C. Umilta \& M. Moscovitch (Eds.), Attention and Performance XV (pp. 589-6I5). Hillsdale, NJ: Erlbaum.

Tanenhaus, M., Spyvey-Knowlton, M. \& Hanna, J. (2000). Modeling thematic and discourse context effects on syntactic ambiguity resolution within a multiple constraints framework: Implications for the architecture of the language processing system. En M. Pickering, C. Clifton \& M. Crocker (Eds.), Architecture and mechanism of the language processing system (pp. 90-I 18). Cambridge: Cambridge University Press.

Trueswell, J. (1996). The role of lexical frequency in syntactic ambiguity resolution. Journal of Memory and Language, 35, 566-585. 
Trueswell, J. (2000). The organization and use of the lexicon for language comprehension. En B. Landau, J. Sabini, J. Jonidis \& E. Newport (Eds.), Perception. cognition and language. Essays in honor of Henry and Lila Gleitman (pp. 327-345). Cambridge, MA: MIT Press.

Trueswell, J., Tanenhaus, M. \& Kello, C. (1993). Verb-specific constraints in sentence processing: Separating effects of lexical preference from garden-paths. Journal of Experimental Psychology: Learning, Memory and Cognition, 19, 528-553.

Trueswell, J. \& Tanenhaus, M. (1994). Toward a lexicalist framework for constraint-based syntactic ambiguity resolution. En C. Clifton, L. Frazier \& K. Rayner (Eds.), Perspectives on sentence processing (Pp. I55- I79). Hillsdale, NJ: Erlbaum.

Trueswell, J., Tanenhaus, M. \& Garnsey, S. (1994). Semantic influences on parsing: Use of thematic role information in syntactic ambiguity resolution. Journal of Memory and Language, 33, 285-3।8.

* Este trabajo ha sido posible gracias al proyecto PSI2009-I3932 financiado por MCYT y dirigido por José Antonio León. 